\title{
Documento: Chlamydia trachomatis: fundamentos de la importancia del cribado en el sistema público de salud
}

\author{
Andrea Huneeus-Vergara7, Humberto Soriano-Brücher², Ricardo Pommer-Tellez ${ }^{3}$, Luis Delpiano-MéndeA, \\ Francisca Salas-Pacheco5, Pablo Céspedes-Pino ${ }^{6}$ y Carolina Schulin-Zeuthen ${ }^{1}$
}

'Presidente Sociedad Chilena de
Obstetricia y Ginecología Infantil
y de la Adolescencia. Comité de
Advocacy de Chlamydia.
PPresidente Sociedad Chilena de
Pediatría.
3Presidente Sociedad Chilena de
Ginecología y Obstetricia.
Presidente Sociedad Chilena de
Infectología.
Presidente Rama de Adolescencia
de Sociedad Chilena de Pediatría.
${ }^{6}$ Presidente. Sociedad Chilena
de Medicina Reproductiva
(SOCMER).
'Directora Sociedad Chilena
de Ginecología Infantil y de
la Adolescencia. Comité de
Advocacy de Chlamydia.
Santiago, agosto de 2018.

Correspondencia a: Andrea Huneeus Vergara andrea.huneeus@gmail.com

\section{Document: Chlamydia trachomatis: fundamentals of the importance of screening in the public health system}

The sexually transmitted infection caused by Chlamydia trachomatis has a high prevalence in Chile and other latitudes and is associated with serious risks to the reproductive health of those who become infected. Screening with self- collected samples and detection by amplification of nucleic acids is the most effective form of sanitary control. Until now, in Chile, the diagnosis and epidemiological management of C. trachomatis is not carried out in public health. The Ministry of Health of Chile is making important progress to start this implementation. The Medical Societies are committed to participate in this process to achieve effective control of this infection in our population.

Keywords: Chlamydia trachomatis; sexually transmitted diseases; screening; self-collection.

Palabras clave: Chlamydia trachomatis; infecciones de transmisión sexual; cribado; auto-toma.

C hlamydia trachomatis representa la infección de transmisión sexual (ITS) de origen bacteriano más frecuente del mundo ${ }^{1}$. La prevalencia global de infección se estima en 1,5\% para personas bajo 50 años de edad; sin embargo, en jóvenes sexualmente activos de 15 a 24 años, esta prevalencia puede llegar a $10 \%{ }^{2}$. Es también un hecho conocido que la prevalencia es mayor en los grupos con mayor desventaja socio-económica ${ }^{3}$.

En Chile, 9\% de los hombres y mujeres sexualmente activos menores de 25 años portan C. trachomatis, según un estudio recientemente publicado ${ }^{4}$. Con datos del Instituto Nacional de Estadísticas-INE, se estima que existen 3.426.529 jóvenes en ese tramo etario, y la encuesta nacional de juventud menciona que $70 \%$ de los jóvenes en ese grupo son sexualmente activos; con esos datos podemos estimar que hay aproximadamente 215.875 jóvenes chilenos infectados que desconocen su estado. Sin embargo, esta reciente publicación de prevalencia y todos los estudios chilenos tienen el sesgo de haber sido realizados en clínicas y centros médicos privados donde está disponible el examen, que atienden a pacientes de nivel socio económico medio o alto ${ }^{4-8}$. La excepción, es una muestra publicada de mujeres embarazadas, adolescentes, del Hospital Padre Hurtado que reportó 19\% de C. trachomatis entre ellas ${ }^{5}$.

Las infecciones por C. trachomatis son, en aproximadamente $80 \%$, asintomáticas. Cuando origina síntomas, produce cuadros de uretritis, proctitis, cervicitis con sangrado post coital, epididimitis, conjuntivitis, y amigdalitis. Cuando la infección se propaga produce enfermedad inflamatoria pélvica, linfogranuloma venéreo, perihepatitis, artritis y neumonía.

Las infecciones no tratadas por C. trachomatis resultan en secuelas reproductivas mayores. En mujeres produce enfermedad inflamatoria pélvica, que genera infertilidad tubaria, embarazos ectópicos y dolor pélvico cróni$\operatorname{co}^{9-11}$. Las mujeres con antecedente de infección por $C$. trachomatis tienen aumentado en $50 \%$ el riesgo de padecer una enfermedad inflamatoria pélvica, $33 \%$ el riesgo de embarazo tubario y $41 \%$ el riesgo de infertilidad tubaria, comparado con quienes no la han tenido ${ }^{12}$. La infección por C. trachomatis aumenta 2,6 veces el riesgo de contagio de VIH y otras ITS, incluido el virus papiloma ${ }^{13-15}$. En mujeres embarazadas, $C$. trachomatis aumenta en $46 \%$ el riesgo de parto prematuro y $50 \%$ el riesgo de rotura prematura de membranas ${ }^{16}$. La transmisión vertical a recién nacidos produce neumonía neonatal y conjuntivitis ${ }^{16,17}$. Existen reportes nacionales que muestran que $18 \%$ de las neumonías neonatales y $8 \%$ de las conjuntivitis neonatales son causadas por C. trachomatis ${ }^{18,19}$.

Dada las altas prevalencia y carga de enfermedad asintomática, el cribado es la mejor manera de prevenir sus secuelas. Cribar una población de mujeres, reduce en $50 \%$ los procesos inflamatorios pélvicos ${ }^{20-23}$. En Europa y Norteamérica, los programas de cribado y la notificación obligatoria de C. trachomatis comenzaron hace ya 15 $\operatorname{años}^{24,25}$. En estos lugares, el cribado se realiza anualmente en todas las mujeres sexualmente activas bajo 25 años de edad, en mujeres embarazadas y en portadores de otra ITS ${ }^{26}$. 
El tratamiento de C. trachomatis es simple, barato y eficaz. Una dosis única de azitromicina de $1 \mathrm{~g}$ basta para mejorar a 95\% de los infectados. Además de evitar las complicaciones, el tratamiento previene la trasmisión y evita la reinfección por parejas sexuales infectadas. Una segunda infección por C. trachomatis aumenta en $20 \%$ el riesgo de complicaciones ${ }^{12}$.

El examen para el diagnóstico de $C$. trachomatis se debe efectuar usando la técnica de amplificación de ácidos nucleicos (reacción de polimerasa en cadena-RPC). Esta técnica tiene una sensibilidad y especificidad cercana a $100 \%$, y permite hacer el diagnóstico con todo tipo de muestras: secreción vaginal, orina, cérvix, hisopado uretral, rectal y faríngea.

Los programas de cribado en sujetos sanos se hacen con auto-toma del examen, esto significa que el propio paciente se toma la muestra en privado y no es necesaria la intervención de un profesional de salud. En hombres, ésta puede ser de orina y en mujeres, de orina y de secreción vaginal. Los estudios de sensibilidad de la auto-toma han demostrado que detectan la misma cantidad de infecciones que el muestreo tradicional. En mujeres, las muestras de auto-toma vaginal pueden detectar incluso más infecciones que las muestras de orina ${ }^{27}$, La auto-toma tiene mayor aceptabilidad especialmente en adolescentes que temen al examen genital ${ }^{29-31}$.

Los avances que están ocurriendo en el Ministerio de Salud de Chile incluyen la solicitud de presupuesto para implementar la RPC de C. trachomatis en el Instituto de Salud Pública para satisfacer la demanda de las muestras de peritaje de abuso sexual a lo largo del país y para establecer los estándares de calidad que se requieran en la futura implementación de la RPC de C. trachomatis en los 25 Servicios de Salud. También está solicitada la codificación FONASA para la RPC de C. trachomatis, que empezará a regir en el arancel del año 2019. Actualmente sólo existe código para la inmunofluorescencia que es un examen obsoleto porque detecta $60 \%$ o menos de las infecciones.

Con los antecedentes expuestos, las Sociedades Médicas científicas aquí firmantes proponen los siguiente:

- Implementar el test diagnóstico de C. trachomatis por RPC con auto-toma en toda la red pública de laboratorios de los Servicios de Salud incluyendo atención primaria y secundaria.

- Crear un programa de tamizaje de C. trachomatis nacional anual para mujeres sexualmente activas bajo 25 años de edad, mujeres embarazadas y cualquier portador de ITS.

- Incorporar la infección por C. trachomatis a las Enfermedades de Notificación Obligatoria.

- Incluir C. trachomatis en la próxima Encuesta Nacional de Salud para hacer un diagnóstico poblacional en todas las regiones y en todos los niveles socio económicos de la infección.

\section{Resumen}

La infección de transmisión sexual por Chlamydia trachomatis, tiene una alta prevalencia en Chile y otras latitudes, y se asocia a graves riesgos para la salud reproductiva de quienes se infectan. El cribado con autotoma de la muestra y detección mediante amplificación de ácidos nucleicos es la forma más eficaz de control sanitario. Hasta ahora, en Chile, el diagnóstico y manejo epidemiológico de C. trachomatis no se realiza en la salud pública. El Ministerio de Salud de Chile está haciendo importantes avances para iniciar esta implementación. La Sociedades Médicas se comprometen a participar en este proceso para lograr el eficaz control de esta infección en nuestra población.

\section{Referencias bibliográficas}

1.- 2014 STD Surveillance /CDC. Available at: http://www.cdc.gov/std/stats14/default.htm. (Consultado el 7 de agosto de 2016).

2.- Datta S D, Torrone E, Kruszon-Moran D, Berman S, Johnson R, Satterwhite C L, et al. Chlamydia trachomatis trends in the United States among persons 14 to 39 years of age, 1999-2008. Sex Transm Dis 2102; 39: 92-6. doi: 10.1097/OLQ.0b013e31823e2ff7.

3.- Crichton J, Hickman M, Campbell R, BatistaFerrer H, Macleod J. Socioeconomic factors and other sources of variation in the prevalence of genital chlamydia infections: A systematic review and meta-analysis. BMC Public Health 2015; 15: 729. doi: 10.1186/s12889-015-20697.
4.- Huneeus A, Schilling A, Fernández M I. Prevalence of Chlamydia trachomatis, Neisseria gonorrhoeae, and Trichomonas vaginalis infection in Chilean adolescents and young adults. J Pediatr Adolesc Gynecol 2018; 31 (4): 411-5. doi:10.1016/j.jpag.2018.01.003.

5.- Huneeus A, Pumarino M G, Schilling A, Robledo P, Bofil M. Prevalencia de Chlamydia trachomatis y Neisseria gonorrhoeae en adolescentes chilenas. Rev Med Chile 2009; 137: 1569-74. doi: /S003498872009001200004

6.- Conejero C, Cannoni G, Merino P M, Bollmann J, Hidalgo C, Castro M, et al. Screening of Neisseria gonorrhoeae and Chlamydia trachomatis using techniques of selfcollected vaginal sample in young women. Rev Chilena Infectol 2013; 30: 489-93. doi: 10.4067/S0716-
10182013000500004.

7.- Romero J, Prado V, Gaete V, Martínez J, Pizarro E. Chlamydia trachomatis infection in asymptomatic Chilean men and with urethritis. Usefulness of first catch urine samples. Rev Med Chile 1997; 125: 1165-71.

8.- Zamboni M, Ralph C, García P, Cuello M. The current prevalence of Chlamydia trachomatis infection among teenagers and young asymptomatic Chilean women justifies the periodic surveillance. Rev Chilena Infectol 2016; 33: 619-27. http://dx.doi.org/10.4067/ S0716-10182016000600003.

9.- Haggerty C L, Gottlieb S L, Taylor B D, Low N, Xu F, Ness R B. Risk of sequelae after Chlamydia trachomatis genital infection in women. J Infect Dis 2010; 201: 134-55. doi: $10.1086 / 652395$. 
10.- Ross J D C. Pelvic inflammatory disease. BMJ Clin Evid 2013; 2013: 1606. https://www. ncbi.nlm.nih.gov/pmc/articles/PMC3859178/ pdf/2013-1606.pdf

11.- Bender N, Herrmann B, Andersen B, Hocking J S, van Bergen J, Morgan J, et al. Chlamydia infection, pelvic inflammatory disease, ectopic pregnancy and infertility: cross-national study. Sex Transm Infect 2011; 87: 601-8. doi: 10.1136/sextrans-2011-050205.

12.- Davies, B. Turner K M E, Frølund M, Ward H, May M T, Rasmussen S, et al. Risk of reproductive complications following chlamydia testing: a population-based retrospective cohort study in Denmark. Lancet Infect Dis 2016; 16: 1057-64. doi: 10.1016/ S1473-3099(16)30092-5.

13.- Fleming D T, Wasserheit J N. From epidemiological synergy to public health policy and practice: the contribution of other sexually transmitted diseases to sexual transmission of HIV infection. Sex Transm Infect 1999; 75 : 3-17.

14.- de Abreu A L, Malaguti N, Souza R P, Uchimura N S, Ferreira E C, Pereira M W, et al. Association of human papillomavirus, Neisseria gonorrhoeae and Chlamydia trachomatis coinfections on the risk of high-grade squamous intraepithelial cervical lesion. Am J Cancer Res 2016; 6: 1371-83. https://www.ncbi.nlm.nih. gov/pmc/articles/PMC4937739/pdf/ajcr00061371.pdf

15.- Reda S, Gonçalves F A, Mazepa M M, Carvalho N S D. Women infected with HIV and the impact of associated sexually transmitted infections. Int J Gynaecol Obstet Off Organ Int Fed Gynaecol Obstet 2018; 142 (2): 143-7. doi:10.1002/ijgo.12507.

16.- Blas M M, Canchihuaman F A, Alva I E, Hawes S E. Pregnancy outcomes in women infected with Chlamydia trachomatis: a population-based cohort study in Washington State. Sex Transm Infect 2007; 83: 314-18. DOI: $10.1136 /$ sti.2006.022665.
17.- Zar H J. Neonatal chlamydial infections: prevention and treatment. Paediatr Drugs 2005; 7: 103-10.

18.- Martínez M A, Millán F, González C. Chlamydia trachomatis genotypes associated with pneumonia in Chilean infants. Scand J Infect Dis 2009; 41: 313-6. doi: 10.1080/00365540902744758.

19.- Valencia O C, Prado J V, Ríos M, Cruz M A, Pilorget J J. Prevalencia de Chlamydia trachomatis en conjuntivitis neonatal determinada mediante las técnicas de inmunofluorescencia y amplificación génica. Rev Med Chile 2000; 128: 758-65. http://dx.doi. org/10.4067/S0034-98872000000700008.

20.- Gottlieb S L, Xu F, Brunham R C. Screening and treating Chlamydia trachomatis genital infection to prevent pelvic inflammatory disease. Sex Transm Dis 2013; 40 (2): 97-102. doi:10.1097/OLQ.0b013e31827bd637.

21.- Low N, Bender N, Nartey L, Shang A, Stephenson J M. Effectiveness of Chlamydia screening: systematic review. Int J Epidemiol 2009; 38: 435-48. doi: 10.1093/ije/dyn222.

22.- Oakeshott P, Kerry S, Aghaizu A, Atherton H, Hay S, Taylor-Robinson D, et al. Randomised controlled trial of screening for Chlamydia trachomatis to prevent pelvic inflammatory disease: the POPI (Prevention of Pelvic Infection) trial. BMJ 2010; 340: c1642. doi: https://doi.org/10.1136/bmj.c164.

23.- van den Broek I V, Hoebe C J, van Bergen J E, Brouwers E E, de Feijter E M, Fennema $\mathrm{J} S$, et al. Evaluation design of a systematic, selective, internet-based, Chlamydia screening implementation in the Netherlands, 20082010: implications of first results for the analysis. BMC Infect Dis 2010; 10: 89. doi: 10.1186/1471-2334-10-8.

24.- Andersen B, Olesen F, Møller J K, Østergaard L. Population-based strategies for outreach screening of urogenital Chlamydia trachomatis infections: A randomized, controlled trial. J Infect Dis 2002; 185: 252-8. DOI: $10.1086 / 33826$.

25.- Kohl K S, Markowitz L E, Koumans E H. Developments in the screening for Chlamydia trachomatis: a review. Obstet. Gynecol. Clin North Am 2003; 30: 637-58. https://doi. org/10.1016/S0889-8545(03)00076-7

26.- CDC-STD Surveillance, 2010. Available at: http://www.cdc.gov/std/stats10/default.htm. (Consultado el 26 de octubre de 2012).

27.- Boyadzhyan B, Yashina T, Yatabe J H, Patnaik M, Hill C S. Comparison of the APTIMA $\mathrm{CT}$ and GC assays with the APTIMA combo 2 assay, the Abbott LCx assay, and direct fluorescent-antibody and culture assays for detection of Chlamydia trachomatis and Neisseria gonorrhoeae. J Clin Microbiol 2004; 42: 3089-93. DOI: 10.1128/JCM.42.7.30893093.2004.

28.- Falk L, Coble B-I, Mjörnberg P-A, Fredlund H. Sampling for Chlamydia trachomatis infection - a comparison of vaginal, firstcatch urine, combined vaginal and firstcatch urine and endocervical sampling. Int J STD AIDS 2010; 21: 283-7 https://doi. org/10.1258\%2Fijsa.2009.009440.

29.- Schachter J, Chernesky M A, Willis D E, Fine P M, Martin D H, Fuller D, et al. Vaginal swabs are the specimens of choice when screening for Chlamydia trachomatis and Neisseria gonorrhoeae: results from a multicenter evaluation of the APTIMA assays for both infections. Sex Transm Dis 2005; 32: 725-8.

30.- Marrazzo J M, Scholes D. Acceptability of urine-based screening for Chlamydia trachomatis in asymptomatic young men: a systematic review. Sex Transm Dis 2008; 35 : S28-33 doi: 10.1097/OLQ.0b013e31816938ca.

31.- Huneeus A, Fernández M I, Schilling A, Parra P, Zakharova A. Adolescents find it easy to collect their own samples to study sexually transmitted infections. Rev Chilena Infectol 2017; 34: 116-9. doi: 10.4067/S071610182017000200003. 